\title{
Sex-Specific Associations of Circulating Uric Acid with Risk of Diabetes Incidence: A Population-Based Cohort Study from Sweden
}

This article was published in the following Dove Press journal: Diabetes, Metabolic Syndrome and Obesity: Targets and Therapy

\author{
Ning Chen (iD) ${ }^{1,2}$ \\ Iram Faqir Muhammad ${ }^{2}$ \\ Zhibin $\mathrm{Li}^{3}{ }^{3}$ \\ Peter M Nilsson (iD) 2,4 \\ Yan Borné ${ }^{2}$ \\ 'Department of Endocrinology, Xiamen \\ Branch, Zhongshan Hospital, Fudan \\ University, Xiamen, People's Republic of \\ China; ${ }^{2}$ Department of Clinical Sciences \\ in Malmö, Lund University, Malmö, \\ Sweden; ${ }^{3}$ Epidemiology Research Unit, \\ The First Affiliated Hospital, Xiamen \\ University, Xiamen, People's Republic of \\ China; ${ }^{4}$ Department of Internal Medicine, \\ Skåne University Hospital, Malmö, \\ Sweden
}

Objective: To explore the longitudinal, as well as sex-specific, associations between circulating uric acid (UA) and diabetes incidence.

Methods: A cohort study of the Malmö Diet Cancer-cardiovascular Cohort (Malmö, Sweden) consisting of 3140 individuals without diabetes at baseline, was followed up until the end of 2018. Incident diabetes cases were identified by linking to local and national diabetes registers. Cox proportional hazard regression was used to assess plasma UA levels in relation to diabetes incidence with adjustment for established confounders.

Results: At baseline, with increasing levels of UA, subjects were more likely to be older and have significantly higher body mass index, waist circumference, triglycerides, C-reactive protein, fasting glucose and 2-h plasma glucose postoral glucose tolerance test, and lower levels of high-density lipoprotein. During a mean follow-up period of $8.09 \pm 2.24$ years, $315(10.0 \%)$ participants developed diabetes, and diabetes incidence rates were $7.89,9.48$ and 18.11 per 1000 person-years for subjects in the 1 st, 2 nd, and 3rd tertiles of UA, respectively (log-rank test: $\mathrm{p}<0.001$ ). With adjustment for potential confounders, elevated UA levels were significantly associated with increased risks of diabetes incidence, with the adjusted hazard ratio (HR) (95\% confidence interval) for per standard deviation increment of UA of $1.22(1.08-1.39, \mathrm{p}=0.002)$. Compared with the $1 \mathrm{st}$ tertile of UA, the 3rd tertile showed significantly increased risk of diabetes incidence with the adjusted HR of $1.74(1.24-2.45, \mathrm{p}=0.002)$, and there was a significant trend between increasing tertiles of UA and diabetes incidence (trend test: $p<0.001$ ). Stratified analyses showed that elevated circulating UA levels were independently associated with increased risks of diabetes incidence in men but not in women, although the interaction between sex and UA was not statistically significant.

Conclusion: Elevated circulating UA was independently associated with increased risk of diabetes incidence, especially for men.

Keywords: cohort study, diabetes incidence, longitudinal, stratified analysis, uric acid

\section{Introduction}

With an estimated 463 million people living with diabetes worldwide, ${ }^{1}$ the cost of treatments for diabetes and its accompanying complications imposes a huge economic burden and represents a major public health problem. Early detection and management are of key importance for prevention and treatment of diabetes. Many risk factors have been established that contribute to the development of diabetes. Some risk factors amenable to modification, such as cigarette smoking, diet and obesity, have been
Correspondence: Yan Borné; Ning Chen Email yan.borne@med.lu.se; ningchen8080@outlook.com
Diabetes, Metabolic Syndrome and Obesity: Targets and Therapy 2020:13 4323-433।

4323

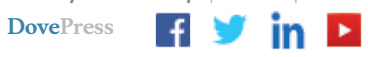

(c) (i) 82020 Chen et al. This work is published and licensed by Dove Medical Press Limited. The full terms of this license are available at https://www.dovepress.com/terms.php cc) you hereby accept the Terms. Non-commercial uses of the work are permitted without any further permission from Dove Medical Press Limited, provided the work is properly attributed. For permission for commercial use of this work, please see paragraphs 4.2 and 5 of our Terms (https://www.dovepress.com/terms.php). 
proven as predictors of diabetes. ${ }^{2}$ However, there is still a need to identify more risk factors for improving risk stratification and for understanding the underlying mechanisms to develop therapeutic interventions. ${ }^{3,4}$

Circulating uric acid (UA) is one such risk factor, already used in clinical practice, which can be a potential predictor for diabetes. UA is a weak acidity heterocyclic compound, a deprotonate durate anion predominantly dissolving in blood. UA can precipitate from blood into tissues by crystalizing into sodium urate as serum UA concentration is higher than $6.8 \mathrm{mg} / \mathrm{dL}$ which is given as its solubility at physiological PH. Using serum UA levels $>7.0 \mathrm{mg} / \mathrm{dL}$ for men and $>5.7 \mathrm{mg} / \mathrm{dL}$ for women as the definition of hyperuricemia, the prevalence rates of hyperuricemia in adults in the United States were $20.2 \%$ and $20.0 \%$, respectively. ${ }^{5}$ It is generally known that hyperuricemia increases the prevalence of gout and uratic nephrolithiasis, while it is also closely related with multiple other chronic conditions, such as obesity, metabolic syndrome, hypertension, hyperlipidemia, fatty liver, chronic kidney disease and cardiovascular disease. ${ }^{6-8}$ Cross-sectional studies have shown that higher UA concentration is associated with higher prevalence of type 2 diabetes. ${ }^{9}$ On the other hand, some studies have reported controversial results, and have not observed an association between UA and increased risk of diabetes. ${ }^{10,11}$ Furthermore, a large study conducted in USA showed inverse relationship between UA and risk of incident diabetes. ${ }^{12}$ Similarly, a study in Bangladesh reported inverse association between observed UA levels and diabetes. ${ }^{13}$ Using a genetic risk score of uric acid, studies using Mendelian randomization analyses did not support a potential causal relationship of serum uric acid levels with incident diabetes risk. ${ }^{14,15}$ Such inconsistencies make it unclear if high $\mathrm{UA}$ is independently associated with the development of diabetes, or not.

Since the sex specific cut-off points for hyperuricemia have been widely used globally, it is of importance to identify if there are sex specific associations between circulating UA and diabetes. However, most of the available evidence in this field are from cross-sectional studies and are controversial. Shani et al reported that serum UA increment was associated with increased risk for diabetes in women but not in men. ${ }^{16}$ Choi et al also found that hyperuricemia was a significantly independent predictor of diabetes only in the female subgroup. ${ }^{17}$ Therefore, there is genuine need to clarify the sex specific associations of circulating UA with diabetes, especially from prospective cohort studies on different ethnic populations.

Therefore, in the present cohort study of 3140 Swedish populations, we firstly aimed to evaluate the longitudinal association between circulating UA level and diabetes incidence. SecondLy, we aimed to explore the potential sex-specific associations of circulating UA level on diabetes incidence.

\section{Methods}

\section{Ethics Statement}

The study was approved by the Lund University Ethical Committee (LU 51/90, LU 2011/537, and LU 2012/762). All participants provided written informed consent.

\section{Study Population}

The Malmö Diet and Cancer cohort is a large prospective population-based cohort comprising of men and women from the city of Malmö in Southern Sweden. Details on subject sampling, recruitment and evaluation have been described in our previous publications. ${ }^{18-20}$ A sub-cohort, the Malmö Diet and Cancer-Cardiovascular cohort (MDCCC) consisting of a random sample of 6103 participants were re-recruited during 1991-1994. ${ }^{19}$ Between May 2007 and September 2012, 3734 individuals from the MDC-CC participated in a re-examination $(76 \%$ attendance of the eligible population). The measurement of plasma UA level was completed in 3668 participants. We excluded 528 participants with a history of prevalent diabetes, leading to a final study population of 3140 subjects.

\section{Measurements}

The re-examination from 2007 to 2012 consisted of a selfadministered questionnaire, physical examination and laboratory tests which have been described previously. ${ }^{15}$ Blood pressure, waist circumference, body mass index (BMI) were measured and calculated from physical examination. Information regarding smoking habits and use of medications was obtained from the questionnaire. Fasting plasma glucose (FPG) was determined using HemoCue (HemoCue AB, Ängelholm, Sweden). Oral glucose tolerance tests (OGTT) after an overnight fast with a measurement of plasma glucose before, and $120 \mathrm{~min}$ after intake of $75 \mathrm{~g}$ of glucose were conducted. High-sensitive $\mathrm{C}$ reactive protein (hs-CRP), triglyceride (TG), total and high density lipoprotein (HDL) cholesterol were measured by standard procedures at the Department of Clinical Chemistry, University Hospital Malmö, Sweden. 
Low density lipoprotein (LDL) cholesterol was calculated by using Friedewald's formula. ${ }^{21}$ UA was measured using Cobas (Roche Diagnostics, USA), with measurement range as: 12-1487 $\mu \mathrm{mol} / \mathrm{L}$.

\section{Ascertainment of Diabetes Incidence}

The first diagnosis of diabetes, emigration from Sweden, death or end of follow-up (December 31st, 2018), whichever came first, were followed-up on each subject. Incident cases of diabetes were retrieved from linkages to several local and national registers. Diabetes was defined as $\mathrm{FPG} \geq 7.0 \mathrm{mmol} / \mathrm{L}$ or a $2 \mathrm{~h}$ post-OGTT plasma glucose $\geq 11.1 \mathrm{mmol} / \mathrm{L}$, which was subsequently verified by a repeated FPG level, selfreport of a physician's diagnosis, or use of antidiabetic medication according to a questionnaire. In order to include only type 2 diabetes, all cases that were specified as type 1 diabetes, secondary diabetes, or others were excluded from the analysis.

\section{Statistical Analysis}

Participants were categorized into sex-specific tertiles according to the concentration of UA levels as well as whether incident diabetes cases or not. The characteristics of the study population across the tertiles of UA or incident diabetes were described as means \pm standard deviations (SD), median (25-75\%) for skewed distribution, or as proportions. The differences were tested using chisquare for categorical variables and analysis of variance (ANOVA) for continuous variables.

Follow-up time until death, emigration, incident diabetes, or end of follow-up was used as time-scale. Incidence rates of diabetes were calculated as incident cases per 1000 person-years of follow-up. Kaplan-Meier curves were used to compare incidence of diabetes across the tertiles of UA for the total sample as well as for men and women separately. The proportional hazard assumptions were tested by incorporating the time-dependent effects of covariates. Cox proportional hazard model was used to estimate the hazard ratios (HR) with $95 \%$ confidence intervals (CI) of UA for incidence of diabetes. Model 1 was adjusted for age and sex; Model 2 was adjusted for age, sex, waist circumference, smoking habits, systolic blood pressure, HDL cholesterol, triglycerides, use of lipid-lowering drugs and fasting glucose. Model 3 was additionally adjusted for CRP levels, the analysis was performed for 2422 subjects due to missing value of CRP. Circulating UA levels were presented as per SD increase as well as tertiles (tertile 2 and tertile 3 vs tertile 1) separately. Trend tests for tertiles of UA were also conducted. Possible interaction between UA levels and sex was investigated further. Stratified analyses of Cox proportional hazard models across sex were further conducted to detect the sex-specific associations of circulating UA with diabetes incidence. Receiver operating characteristic (ROC) curve was used to illustrate the prediction of the incidence of diabetes. In a sub-analysis BMI was adjusted instead of waist as it has shown to be an important confounder in the relationship between diabetes and UA. ${ }^{22}$

A $p$-value of less than 0.05 was regarded as statistically significant. All analyses were performed using IBM SPSS Statistics version 24 (IBM Corp, Armonk, New York, USA).

\section{Results}

\section{Baseline Characteristics of Participants}

The baseline characteristics of the study participants across tertiles of UA (mean ( \pm SD): $233.74( \pm 35.88), 297.55$ $( \pm 31.09)$ and $383.45( \pm 59.37)(\mu \mathrm{mol} / \mathrm{L})$, respectively) as well as whether they were incident diabetes cases during the follow-up are presented in Table 1. Generally, with increasing levels of UA, subjects were more likely to be older, had significantly higher BMI, waist circumference, triglycerides, CRP, fasting glucose and $2 \mathrm{~h}$ post-OGTT, but showed lower levels of HDL cholesterol. The individuals who developed diabetes during the follow up generally showed significantly increased baseline levels of BMI, waist circumference, systolic blood pressure, triglycerides, CRP, fasting glucose, and $2 \mathrm{~h}$ post-OGTT, but had significantly decreased HDL-cholesterol. Furthermore, incident diabetes cases also showed significantly increased circulating UA than the controls $(328.34 \pm 77.73$ vs $302.21 \pm 74.56$ $\mu \mathrm{mol} / \mathrm{L}, \mathrm{p}<0.001)$.

\section{Incidence Rates of Diabetes Stratified by Tertiles of UA and Sex}

Table 2 showed the incidence rates of diabetes stratified by tertiles of circulating uric acid for all the subjects as well as for men and women separately. For all subjects, during a mean follow up period of $8.09 \pm 2.24$ years, a total of 315 $(10.0 \%)$ participants developed diabetes. The incidence rates of diabetes were $7.89,9.48$ and 18.11 per 1000 person-years for subjects in the 1st, 2nd and 3rd tertile of UA, respectively. The cumulative incidence of diabetes across the tertiles of UA for all the subjects is presented in Figure 1A, and subjects in the 3rd tertile of UA had significantly the highest incidence rate of diabetes (log-rank test: $\mathrm{p}<0.001)$. The cumulative 
Table I Baseline Characteristics Stratified by the Tertiles of Uric Acid Level and Incident Diabetes $(n=3 / 40)$

\begin{tabular}{|c|c|c|c|c|c|c|c|}
\hline \multirow[t]{2}{*}{ Variables } & \multicolumn{4}{|c|}{ Baseline Uric Acid } & \multicolumn{3}{|c|}{ Incident Diabetes } \\
\hline & Tertile I & Tertile 2 & Tertile 3 & P-value & Yes & No & P-value \\
\hline N (\%) & 1043 (33.2\%) & 1056 (33.6\%) & 1041 (33.2\%) & & $315(10.0 \%)$ & $2825(90.0 \%)$ & \\
\hline Age (years) & $71.44 \pm 5.54$ & $72.36 \pm 5.56$ & $73.21 \pm 5.64$ & $<0.001$ & $71.84 \pm 5.85$ & $72.39 \pm 5.60$ & 0.101 \\
\hline Current smoker (\%) & $12.3 \%$ & $10.0 \%$ & $7.6 \%$ & $<0.001$ & $10.7 \%$ & $9.8 \%$ & 0.123 \\
\hline BMI $\left(\mathrm{kg} / \mathrm{m}^{2}\right)$ & $25.16 \pm 3.78$ & $26.42 \pm 3.80$ & $28.10 \pm 4.50$ & $<0.001$ & $28.53 \pm 4.70$ & $26.34 \pm 4.10$ & $<0.001$ \\
\hline Waist circumference $(\mathrm{cm})$ & $87.61 \pm 11.59$ & $90.97 \pm 11.48$ & $95.30 \pm \mid 1.91$ & $<0.001$ & $97.45 \pm 12.10$ & $90.61 \pm 11.88$ & $<0.001$ \\
\hline Systolic blood pressure $(\mathrm{mmHg})$ & $|42.2| \pm 19.14$ & $|42.9| \pm \mid 8.65$ & $143.04 \pm 19.02$ & 0.317 & $147.13 \pm 20.06$ & $142.23 \pm 18.75$ & $<0.001$ \\
\hline Use of lipid lowering drugs, \% & $19.6 \%$ & $25.6 \%$ & $33.4 \%$ & $<0.001$ & $36.2 \%$ & $25.1 \%$ & $<0.001$ \\
\hline Use of ACE inhibitors (\%) & $14.2 \%$ & $18.8 \%$ & $30.5 \%$ & $<0.001$ & $25.1 \%$ & $20.7 \%$ & 0.072 \\
\hline Triglyceride (mmol/L) & $0.91 \pm 0.39$ & $1.05 \pm 0.57$ & $1.23 \pm 0.58$ & $<0.001$ & $1.27 \pm 0.60$ & $1.04 \pm 0.52$ & $<0.001$ \\
\hline HDL-cholesterol (mmol/L) & $1.54 \pm 0.44$ & $1.46 \pm 0.45$ & $1.35 \pm 0.39$ & $<0.001$ & $1.29 \pm 0.38$ & $1.47 \pm 0.44$ & $<0.001$ \\
\hline LDL-cholesterol (mmol/L) & $3.42 \pm 0.87$ & $3.40 \pm 0.90$ & $3.33 \pm 0.97$ & 0.026 & $3.2 I \pm 0.87$ & $3.40 \pm 0.92$ & 0.001 \\
\hline 0-h PG (OGTT, mmol/L) & $5.72 \pm 0.76$ & $5.79 \pm 0.67$ & $5.95 \pm 0.74$ & $<0.001$ & $6.70 \pm 1.10$ & $5.72 \pm 0.60$ & $<0.001$ \\
\hline 2-h PG (OGTT, mmol/L) & $6.67 \pm 2.05$ & $6.90 \pm 2.97$ & $7.56 \pm 2.37$ & $<0.001$ & $10.05 \pm 4.74$ & $6.67 \pm 1.87$ & $<0.001$ \\
\hline \multirow[t]{2}{*}{ CRP (mg/L) (median (P25-P75))* } & 1.10 & 1.40 & 2.10 & $<0.001$ & 2.10 & 1.40 & $<0.001$ \\
\hline & $(0.10-2.30)$ & $(0.70-2.80)$ & $(0.97-3.90)$ & & $(1.00-4.10)$ & $(0.68-2.90)$ & \\
\hline Uric acid $(\mu \mathrm{mol} / \mathrm{L})$ & $233.74 \pm 35.88$ & $297.55 \pm 31.09$ & $383.45 \pm 59.37$ & $<0.001$ & $328.34 \pm 77.73$ & $302.21 \pm 74.56$ & $<0.001$ \\
\hline
\end{tabular}

Notes: *Median (P25-P75) is presented for CRP. All other values were means \pm standard deviation or percentages (\%).

Abbreviations: BMI, body mass index; CRP, C-reactive protein; HDL, high-density lipoprotein; LDL, low-density lipoprotein cholesterol; OGTT, oral glucose tolerance test; UA, uric acid.

incidence rates of diabetes across the sex-specific tertiles of UA for men and women are presented in Figure 1B and C, respectively. Similar to all the subjects, the sex-specific 3rd tertile of UA for both men and women showed significantly the highest incidence rate of diabetes (llog-rank test: $\mathrm{p}<0.001)$.

\section{Association of circulating UA on Diabetes Incidence for All the Subjects}

Cox proportional hazard regression was conducted to estimate the independent effect of circulating UA on diabetes incidence with adjustment for potential confounders. In Model 1 with adjustment for sex and age, subjects in the tertile 3 had significantly higher risk of developing diabetes as compared to those in the tertile 1 (HR: $2.30 .95 \%$ CI: 1.74-3.05; P for trend $<0.001$ ) (Table 2). In Model 2, after additional adjustments for waist circumference, smoking habits, systolic blood pressure, HDL cholesterol, triglycerides, use of lipid-lowering drugs and fasting glucose, the risk of incident diabetes was attenuated was still statistically significant for those in tertile 3 vs tertile 1 (HR: $1.79,95 \%$ CI: 1.30-2.47; P for trend: $<0.001)$. After further adjustment for CRP in Model 3, the HR for diabetes in tertile 3 compared with the tertile 1 was 1.74 (95\% CI: 1.24-2.45; P for trend: $<0.001)$. For per 1- SD increment of UA, the adjusted HR were 1.22 (95\% CI: 1.08-1.39). The ROC curve for incident diabetes with UA and all the potential confounders is shown in Figure 2. The area under the ROC curve was 0.838 (95\% CI: $0.810-0.866$ ).

In the sub-analysis, when the final Cox model was adjusted for BMI instead of waist in the whole cohort, the results were very similar with the adjusted HR of 1.79 $(1.27-2.52, \mathrm{p}=0.001)$ (the $3 \mathrm{rd}$ tertile compared with the $1 \mathrm{st}$ tertile of UA).

\section{Sex-Specific Associations of Circulating UA with Diabetes Incidence}

Although the interaction test between sex and circulating UA on diabetes incidence was not statistically significant $(\mathrm{p}>0.05)$, Cox proportional hazard regression analyses stratified by sex were conducted to explore the sexspecific independent effects of circulating UA on diabetes incidence with adjustment for the potential confounders. For men, Table 3 (Model 3) showed that higher circulating UA levels were still significantly associated with higher risks of diabetes incidence, and the adjusted HR with $95 \%$ CI for per SD increase of UA was 1.21 (1.01-1.47, $\mathrm{p}=0.046$ ). However, increasing tertiles of UA did not show significantly independent effect on diabetes incidence (trend test: $\mathrm{p}=0.145$ ). For women, increasing circulating UA level was not statistically significant associated with diabetes incidence (trend test: $\mathrm{p}=0.199$ ). 
Table 2 Incidence Rate (1/1000 Person-Years) of Diabetes Stratified by Sex-Specific Tertiles of Baseline Uric Acid $(n=3 \mid 40)$

\begin{tabular}{|c|c|c|c|c|}
\hline \multirow[t]{2}{*}{$\begin{array}{l}\text { Baseline Uric } \\
\text { Acid }\end{array}$} & \multirow[t]{2}{*}{$\mathbf{N}$} & \multirow[t]{2}{*}{$\begin{array}{l}\text { Person- } \\
\text { Years }\end{array}$} & \multirow{2}{*}{$\begin{array}{l}\text { Number } \\
\text { of } \\
\text { Incident } \\
\text { Diabetes }\end{array}$} & $\begin{array}{l}\text { Diabetes } \\
\text { Incidence } \\
\text { Rate }\end{array}$ \\
\hline & & & & $\begin{array}{l}\text { ( } 1 / 1000 \\
\text { Person- } \\
\text { Years) }\end{array}$ \\
\hline \multicolumn{5}{|l|}{ Total $(n=3 \mid 40)$} \\
\hline \multicolumn{5}{|l|}{ Tertiles of UA } \\
\hline $\begin{array}{l}\text { Tertile I } \\
\text { Tertile } 2 \\
\text { Tertile } 3 \\
\text { P-value }\end{array}$ & $\begin{array}{l}1043 \\
1056 \\
1041\end{array}$ & $\begin{array}{l}9120.99 \\
9068.91 \\
8671.69\end{array}$ & $\begin{array}{l}72 \\
86 \\
157\end{array}$ & $\begin{array}{l}7.89 \\
9.48 \\
18.11 \\
<0.001\end{array}$ \\
\hline \multicolumn{5}{|l|}{ Men $(n=1227)$} \\
\hline \multicolumn{5}{|l|}{ Tertiles of UA } \\
\hline $\begin{array}{l}\text { Tertile } 1 \\
\text { Tertile } 2 \\
\text { Tertile } 3 \\
\text { P-value }\end{array}$ & $\begin{array}{l}412 \\
409 \\
406\end{array}$ & $\begin{array}{l}3527.01 \\
3397.50 \\
3327.56\end{array}$ & $\begin{array}{l}40 \\
35 \\
63\end{array}$ & $\begin{array}{l}11.34 \\
10.30 \\
18.93 \\
<0.001\end{array}$ \\
\hline \multicolumn{5}{|c|}{ Women $(n=19 \mid 3)$} \\
\hline \multicolumn{5}{|l|}{ Tertiles of UA } \\
\hline $\begin{array}{l}\text { Tertile I } \\
\text { Tertile } 2 \\
\text { Tertile } 3 \\
\text { P-value }\end{array}$ & $\begin{array}{l}631 \\
647 \\
635\end{array}$ & $\begin{array}{l}5593.98 \\
5671.41 \\
5344.13\end{array}$ & $\begin{array}{l}32 \\
51 \\
94\end{array}$ & $\begin{array}{l}5.72 \\
8.99 \\
17.59 \\
<0.001\end{array}$ \\
\hline
\end{tabular}

Abbreviation: UA, uric acid.

\section{Discussion}

Based on the Malmö Diet and Cancer-Cardiovascular Cohort (MDC-CC) of 3140 population-based study, we found that increasing levels of UA were significantly associated with higher levels of age, BMI, waist circumference, triglycerides, CRP, fasting glucose and $2 \mathrm{~h}$ postOGTT but lower level of HDL cholesterol at baseline. After a mean duration of $8.09 \pm 2.24$ years of follow-up, 315 subjects developed diabetes and showed significantly higher baseline circulating UA levels than their controls. Kaplan-Meier survival curves showed that the 3rd tertile of UA showed significantly the highest incidence rate of diabetes for all subjects as well as for men and women, separately. After adjusting for all the potential confounding factors, we found that elevated circulating UA levels were significantly associated with increased risks of diabetes incidence. Compared with 1st tertile of UA, the 3rd tertile showed significantly increased risk of diabetes incidence and there was a significant trend between increasing tertiles of circulating UA and diabetes incidence. Although there was no significant interaction between sex and UA on diabetes incidence, stratified analyses for men and women showed that higher circulating UA levels were independently associated with increased risks of diabetes incidence for men but not for women.

In recent years, emerging evidence has suggested that high levels of UA may be a risk factor for developing diabetes. Results from meta-analysis studies have shown that the risk of diabetes was increased by $6-11 \%$ for every $1 \mathrm{mg} / \mathrm{dL}$ increase in UA. ${ }^{9,23,24}$ A cohort study in US examined the risk of new-onset diabetes among 1923 male veterans with gout and no previous history of diabetes and found that hyperuricemia was associated with a significantly higher risk of developing diabetes. ${ }^{25}$ Overall, these findings are in accordance with the hypothesis that the UA and diabetes incidence are closely related. UA has also been shown to be closely associated with glucose metabolism and metabolic syndrome. ${ }^{26}$ It can be speculated that UA may play a role in the pathophysiology of diabetes and experimental researches have suggested that oxidative stress maybe the major mechanism. As a pro-oxidant, high UA levels increase reactive oxygen species (ROS), whose overproduction may be also caused by Xanthine oxidoreductase (XO) expression and activity. XO catalyzes the final two reactions in the biochemical chain that leads to UA formation. The second step of this process is responsible for the superoxide anion radical and/or hydrogen peroxide $\mathrm{H}_{2} \mathrm{O}_{2}$ formation. ${ }^{27,28} \mathrm{UA}$ may enhance production of ROS and can lead to the loss of transcription factors needed for insulin gene expression, leading to decreased insulin production and secretion. ${ }^{29}$ Experimental studies demonstrated that elevated levels of UA cause $\beta$-cell injury via the NF-kB-iNOS-NO signaling axis. $^{30}$ High levels of UA may also induce inflammatory pathways by activating p38 MAPK and NF- $\kappa$ B in vascular smooth muscle cells, ${ }^{31}$ contributing to insulin resistance in the liver by inducing mitochondrial oxidative stress and steatosis, ${ }^{32}$ induce local inflammation in the adipose tissue with a reduction in the production of adiponectin, ${ }^{33}$ and reduce the ability of insulin to stimulate vasodilation of blood vessels, which is important for the delivery of glucose to the skeletal muscle. ${ }^{34}$ This leads to increased insulin resistance and impaired insulin secretion, eventually leading to type 2 diabetes. Unfortunately, fasting insulin levels were not checked in the individuals of our cohort, and homeostasis model assessment - insulin resistance (HOMA-IR) and HOMA- $\beta$ could not be calculated. 


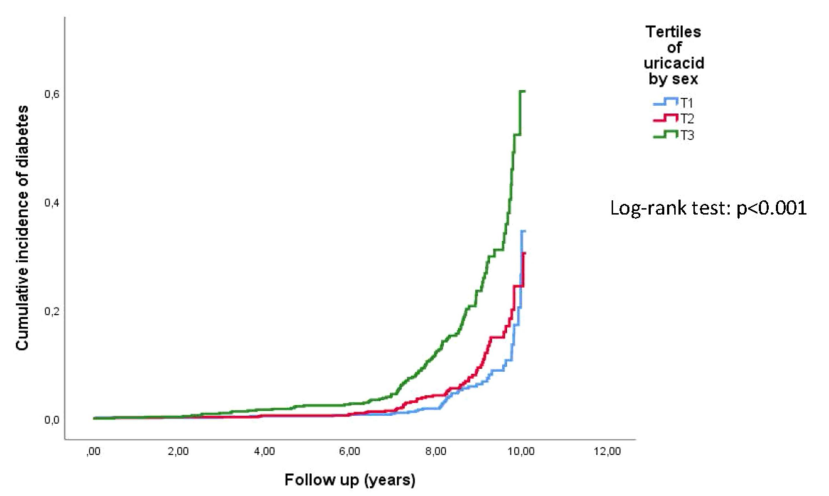

A

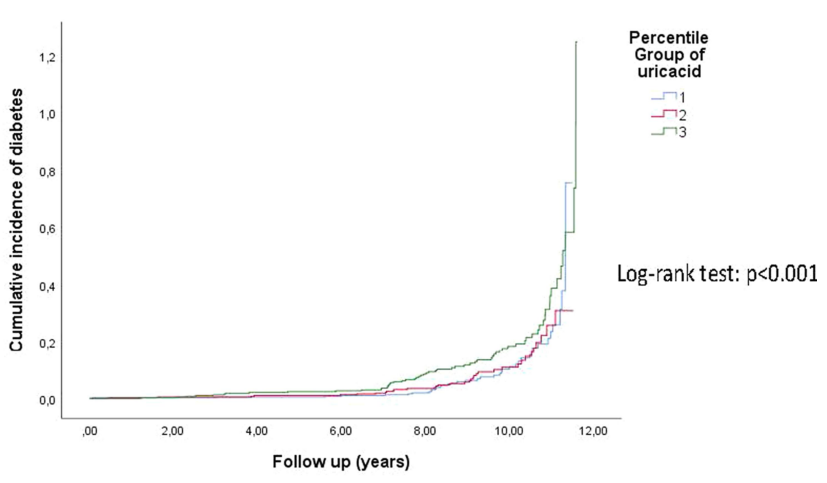

B

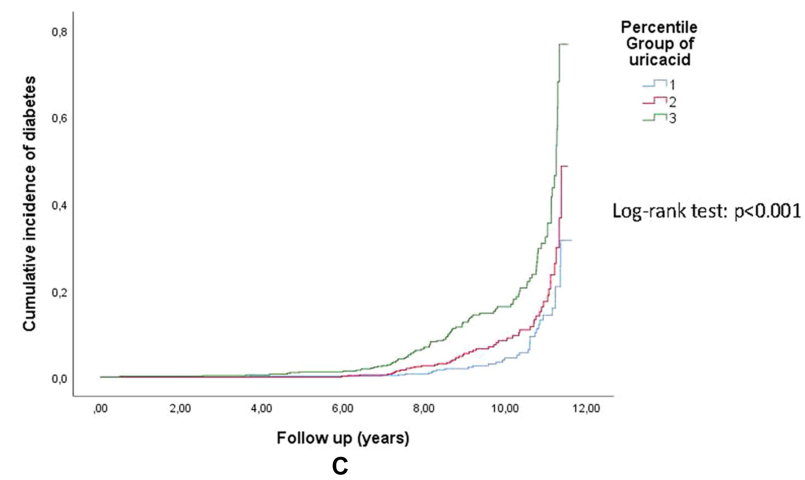

Figure I (A) Cumulative incidence of diabetes across the tertiles of baseline uric acid in total sample. (B) Cumulative incidence of diabetes in relation to the tertiles of baseline uric acid in men. (C) Cumulative incidence of diabetes in relation to the tertiles of baseline uric acid in women.

Results on sex-specific associations between circulating UA and diabetes incidence from different populations have been controversial. Shani et al reported sex-stratified

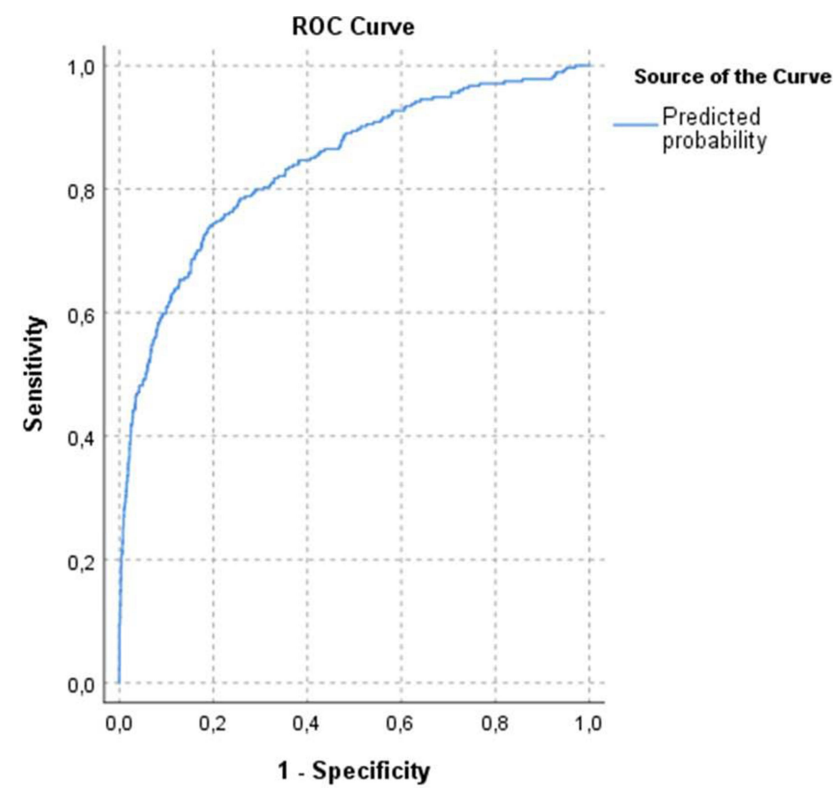

Figure 2 Receiver operating characteristic (ROC) curve of the prediction of diabetes incidence. results in their study of 30,302 healthy participants with normal weight and they found that a $1-\mathrm{mg} / \mathrm{dL}$ serum UA increment was associated with increased risk for diabetes in women but not in men. ${ }^{16}$ A retrospective study consisting of 10,505 participants enrolled at a cardiovascular center without history of diabetes at baseline also found that the risk of diabetes increased when UA increased only in the female subgroup. ${ }^{17}$ Although the present study did not find a significant interaction between sex and UA on diabetes incidence, stratified analyses across sex showed that higher circulating UA levels were independently associated with increased risks of diabetes incidence in men but not in women, which were controversial with the previous two reports above. The possible reasons for the conflicting findings between ours and others may be due to: the diabetes incidence rate in men in the present study was relatively higher than that in women as shown in Table 2.

There are some strengths of the present study. It is a population-based study with a prospective design. Individuals with previous history of diabetes were carefully excluded from the study. The diabetes incidence rate in the present study (around 11 per 1000 person-years) 
Table 3 Adjusted Hazards Ratios (HRs) with Associated 95\% Confidence Interval (Cl) for Incident Diabetes in Relation to Baseline Circulating Uric Acid

\begin{tabular}{|c|c|c|c|c|c|c|c|c|}
\hline \multirow[t]{3}{*}{ Variables } & \multicolumn{2}{|c|}{ Baseline Uric Acid $^{\dagger}$} & \multicolumn{6}{|c|}{ Tertiles of Baseline Uric Acid } \\
\hline & \multirow[t]{2}{*}{ HR (95\% Cl) } & \multirow[t]{2}{*}{ P-value } & \multirow{2}{*}{$\begin{array}{l}\text { Tertile I } \\
\text { HR }\end{array}$} & \multicolumn{2}{|l|}{ Tertile 2} & \multicolumn{2}{|l|}{ Tertile 3} & \multirow[t]{2}{*}{$P$ value for Trend Test } \\
\hline & & & & HR (95\% Cl) & $P$ value & HR (95\% Cl) & $P$ value & \\
\hline \multicolumn{9}{|l|}{ Total } \\
\hline Model I $(n=3 \mid 40)$ & $1.38(1.23-1.54)$ & $<0.001$ & 1.00 & $1.30(0.95-1.78)$ & 0.100 & $2.30(1.74-3.05)$ & $<0.001$ & $<0.001$ \\
\hline Model $2(n=3 \mid 40)$ & $1.22(1.08-1.37)$ & 0.002 & 1.00 & $1.21(0.86-1.70)$ & 0.270 & $1.79(1.30-2.47)$ & $<0.001$ & $<0.001$ \\
\hline Model $3(n=2422)$ & $1.22(1.08-1.39)$ & 0.002 & 1.00 & $1.14(0.79-1.64)$ & 0.500 & $1.74(1.24-2.45)$ & 0.002 & $<0.001$ \\
\hline \multicolumn{9}{|l|}{ Men } \\
\hline Model I $(n=1227)$ & $1.26(1.07-1.49)$ & 0.005 & 1.00 & $0.98(0.62-1.55)$ & 0.931 & $1.49(0.99-2.22)$ & 0.055 & 0.045 \\
\hline Model $2(n=1227)$ & $1.26(1.05-1.50)$ & 0.013 & 1.00 & $0.99(0.61-1.64)$ & 0.991 & $1.46(0.93-2.30)$ & 0.100 & 0.077 \\
\hline Model $3(n=949)$ & $1.21(1.01-1.47)$ & 0.046 & 1.00 & $0.94(0.55-1.62)$ & 0.835 & $1.40(0.85-2.29)$ & 0.185 & 0.145 \\
\hline \multicolumn{9}{|l|}{ Women } \\
\hline Model I $(n=1913)$ & $1.49(1.29-1.73)$ & $<0.001$ & 1.00 & $2.30(1.74-3.05)$ & 0.022 & $3.42(2.28-5.13)$ & $<0.001$ & $<0.001$ \\
\hline Model $2(n=|9| 3)$ & $0.99(0.82-1.18)$ & 0.882 & 1.00 & $0.92(0.55-1.54)$ & 0.742 & $1.25(0.76-2.04)$ & 0.382 & 0.219 \\
\hline Model $3(n=1473)$ & $1.04(0.85-1.26)$ & 0.722 & 1.00 & $0.92(0.55-1.53)$ & 0.742 & $1.25(0.76-2.04)$ & 0.382 & 0.199 \\
\hline
\end{tabular}

Notes: ${ }^{\dagger} \mathrm{Hazards}$ ratios (HR) and $95 \%$ confidence interval $(\mathrm{Cl})$ was expressed by per SD increase of serum uric acid. ${ }^{\ddagger} \mathrm{HR}$ and $95 \% \mathrm{Cl}$ was expressed by the first tertile of serum uric acid as the reference. Model I: Adjusted for age and sex. Model 2: Model I plus waist circumference, smoking habits, systolic blood pressure, HDL cholesterol, triglycerides, use of lipid lowering drugs and fasting glucose. Model 3: Model 2 plus C-reactive protein.

was quite similar to that of adults with the age $\geq 65$ years old in the United States. ${ }^{35}$ All new cases were identified through linkages with validated local and national registers. In our regression analyses of the incidence of diabetes, the potential confounding risk factors, including CRP level which has been shown to be linked with both hyperuricemia and diabetes, ${ }^{36,37}$ have been adjusted for in the present study. Some limitations also need to be acknowledged. Data of the current study was from a cohort consisting of mainly Caucasian subjects carried out in Malmö, Sweden, which limits the generalizability of the results. Another limitation is the lack of serial measurements of UA and risk factors during the followup. However, we had adjusted for potential confounders, and the results were largely independent of these variables. Moreover, since this is an observational study, residual confounding bias cannot be ruled out.

\section{Conclusion}

In conclusion, this observational cohort study demonstrated that elevated plasma UA levels were significantly associated with higher risks of developing diabetes, independent of established risk factors, and there was a significant trend between increasing tertiles of circulating UA and diabetes incidence. Therefore, screening and treatment for high plasma UA are important to prevent diabetes incidence. Furthermore, controversial with previous findings on the sex specific associations of circulating UA with diabetes, we found that higher circulating UA levels were independently associated with increased risks of diabetes incidence only in men but not in women. Therefore, more prospective cohort studies are warranted to clarify the sex-specific relationship between UA and diabetes incidence in future.

\section{Ethical Approval}

All procedures performed in studies involving human participants were in accordance with the ethical standards of the institutional and/or national research committee and with the Helsinki Declaration and its later amendments or comparable ethical standards.

\section{Acknowledgments}

The authors acknowledge the information provided by the National Diabetes Register of Sweden, the MHR, the Diabetes 2000 register, and the registers provided by the Swedish Board of Health and Welfare. The authors wish to thank all participants in the Malmö Diet and Cancer Study for making this study possible. The approval for research from this database is obtained through the Malmö Diet and Cancer Study (MDCS) steering committee. Data are 
available upon request for interested researchers by applying to MDCS steering committee (Anders.Dalin@med.lu. se) with ethical approval.

\section{Author Contributions}

All authors made a significant contribution to the work reported, whether that is in the conception, study design, execution, acquisition of data, analysis and interpretation, or in all these areas; took part in drafting, revising or critically reviewing the article; gave final approval of the version to be published; have agreed on the journal to which the article has been submitted; and agree to be accountable for all aspects of the work.

\section{Funding}

This study was supported by grants from the Research Council of Sweden to PMN, as well as from Swedish Heart and Lung foundation, and ALF of the county council.

\section{Disclosure}

The authors declare that they have no conflicting interest.

\section{References}

1. Federation ID. IDF Diabetes Atlas. 9th. Brussels, Belgium; 2019.

2. Imamura F, Mukamal KJ, Meigs JB, et al. Risk factors for type 2 diabetes mellitus preceded by beta-cell dysfunction, insulin resistance, or both in older adults: the Cardiovascular Health Study. Am J Epidemiol. 2013;177(12):1418-1429. doi:10.1093/aje/kws440

3. Glumer C, Vistisen D, Borch-Johnsen K, Colagiuri S, Collaboration D. Risk scores for type 2 diabetes can be applied in some populations but not all. Diabetes Care. 2006;29:410-414. doi:10.2337/diacare.29.02.06.dc05-0945

4. Noble D, Mathur R, Dent T, Meads C, Greenhalgh T. Risk models and scores for type 2 diabetes: systematic review. BMJ. 2011;343(nov28 1):d7163. doi:10.1136/bmj.d7163

5. Chen-Xu M, Yokose C, Rai SK, Pillinger MH, Choi HK. Contemporary prevalence of gout and hyperuricemia in the United States and decadal trends: the National Health and Nutrition Examination Survey, 2007-2016. Arthritis Rheumatol. 2019;71:991-999. doi:10.1002/art.40807

6. Sattui SE, Singh JA, Gaffo AL. Comorbidities in patients with crystal diseases and hyperuricemia. Rheum Dis Clin North Am. 2014;40 (2):251-278. doi:10.1016/j.rdc.2014.01.005

7. De Pergola G, Cortese F, Termine G, et al. Uric acid, metabolic syndrome and atherosclerosis: the chicken or the egg, which comes first? Endocr Metab Immune Disord Drug Targets. 2018;18:251-259. doi:10.2174/1871530318666180212101548

8. Zupo R, Castellana F, Boninfante B, et al. Uric acid and potassium serum levels are independent predictors of blood pressure non-dipping in overweight or obese subjects. Nutrients. 2019;11(12):2970. doi:10.3390/nu11122970

9. Mortada I. Hyperuricemia, type 2 diabetes mellitus, and hypertension: an emerging association. Curr Hypertens Rep. 2017;19:69.
10. Li X, Meng X, Gao X, et al. Elevated serum xanthine oxidase activity is associated with the development of type 2 diabetes: a Prospective Cohort Study. Diabetes Care. 2018;41:884-890.

11. Van der Schaft N, Brahimaj A, Wen K-X, Franco OH, Dehghan A, Hribal ML. The association between serum uric acid and the incidence of prediabetes and type 2 diabetes mellitus: the Rotterdam Study. PLoS One. 2017;12(6):e0179482. doi:10.1371/journal. pone. 0179482

12. Bandaru P, Shankar A. Association between serum uric acid levels and diabetes mellitus. Int $J$ Endocrinol. 2011;2011:604715. doi:10.1155/2011/604715

13. Haque T, Rahman S, Islam S, Molla NH, Ali N. Assessment of the relationship between serum uric acid and glucose levels in healthy, prediabetic and diabetic individuals. Diabetol Metab Syndr. 2019;11:49.

14. Keerman M, Yang F, Hu H, et al. Mendelian randomization study of serum uric acid levels and diabetes risk: evidence from the Dongfeng-Tongji cohort. BMJ Open Diabetes Res Care. 2020;8: e000834.

15. Sluijs I, Holmes MV, van der Schouw YT, et al. A mendelian randomization study of circulating uric acid and type 2 diabetes. Diabetes. 2015;64(8):3028-3036. doi:10.2337/db14-0742

16. Shani M, Vinker S, Dinour D, et al. High normal uric acid levels are associated with an increased risk of diabetes in lean, normoglycemic healthy women. $J$ Clin Endocrinol Metab. 2016;101(10):3772-3778. doi:10.1210/jc.2016-2107

17. Choi BG, Kim DJ, Baek MJ, et al. Hyperuricaemia and development of type 2 diabetes mellitus in Asian population. Clin Exp Pharmacol Physiol. 2018;45:499-506. doi:10.1111/1440-1681.12911

18. Berglund G, Elmstahl S, Janzon L, Larsson SA. The Malmo Diet and Cancer Study. Design and feasibility. J Intern Med. 1993;233:45-51. doi:10.1111/j.1365-2796.1993.tb00647.x

19. Hedblad B, Nilsson P, Janzon L, Berglund G. Relation between insulin resistance and carotid intima-media thickness and stenosis in non-diabetic subjects. Results from a cross-sectional study in Malmo, Sweden. Diabet Med. 2000;17(4):299-307. doi:10.1046/j.14645491.2000.00280.x

20. Rosvall M, Persson M, Ostling G, et al. Risk factors for the progression of carotid intima-media thickness over a 16-year follow-up period: the Malmö Diet and Cancer Study. Atherosclerosis. 2015;239(2):615-621. doi:10.1016/j.atherosclerosis.2015.01.030

21. Friedewald WT, Levy RI, Fredrickson DS. Estimation of the concentration of low-density lipoprotein cholesterol in plasma, without use of the preparative ultracentrifuge. Clin Chem. 1972;18(6):499-502. doi:10.1093/clinchem/18.6.499

22. Ali N, Perveen R, Rahman S, et al. Prevalence of hyperuricemia and the relationship between serum uric acid and obesity: a study on Bangladeshi adults. PLoS One. 2018;13(11):e0206850. doi:10.1371/ journal.pone. 0206850

23. Kodama S, Saito K, Yachi Y, et al. Association between serum uric acid and development of type 2 diabetes. Diabetes Care. 2009;32 (9):1737-1742. doi:10.2337/dc09-0288

24. Lv Q, Meng X-F, He -F-F, et al. High serum uric acid and increased risk of type 2 diabetes: a systemic review and meta-analysis of prospective cohort studies. PLoS One. 2013;8(2):e56864. doi:10.1371/journal.pone. 0056864

25. Krishnan E, Akhras KS, Sharma H, et al. Relative and attributable diabetes risk associated with hyperuricemia in US veterans with gout. QJM. 2013;106(8):721-729. doi:10.1093/qjmed/hct093

26. Soltani Z, Rasheed K, Kapusta DR, Reisin E. Potential role of uric acid in metabolic syndrome, hypertension, kidney injury, and cardiovascular diseases: is it time for reappraisal? Curr Hypertens Rep. 2013;15(3):175-181. doi:10.1007/s11906-013-0344-5

27. Harrison R. Structure and function of xanthine oxidoreductase: where are we now? Free Radic Biol Med. 2002;33(6):774-797. doi:10.1016/ S0891-5849(02)00956-5 
28. Harrison R. Physiological roles of xanthine oxidoreductase. Drug Metab Rev. 2004;36(2):363-375. doi:10.1081/DMR-120037569

29. Matsuoka T, Kajimoto Y, Watada H, et al. Glycation-dependent, reactive oxygen species-mediated suppression of the insulin gene promoter activity in HIT cells. $J$ Clin Invest. 1997;99:144-150. doi:10.1172/JCI119126

30. Jia L, Xing J, Ding Y, et al. Hyperuricemia causes pancreatic beta-cell death and dysfunction through NF-kappaB signaling pathway. PLoS One. 2013;8:e78284. doi:10.1371/journal.pone. 0078284

31. Kanellis J, Watanabe S, Li JH, et al. Uric acid stimulates monocyte chemoattractant protein-1 production in vascular smooth muscle cells via mitogen-activated protein kinase and cyclooxygenase- 2 . Hypertension. 2003;41:1287-1293. doi:10.1161/01.HYP.0000072 820.07472.3B

32. Lanaspa MA, Sanchez-Lozada LG, Choi YJ, et al. Uric acid induces hepatic steatosis by generation of mitochondrial oxidative stress: potential role in fructose-dependent and -independent fatty liver. J Biol Chem. 2012;287:40732-40744. doi:10.1074/jbc.M112.399899
33. Baldwin W, McRae S, Marek G, et al. Hyperuricemia as a mediator of the proinflammatory endocrine imbalance in the adipose tissue in a murine model of the metabolic syndrome. Diabetes. 2011;60:1258-1269. doi:10.2337/db10-0916

34. Sanchez-Lozada LG, Lanaspa MA, Cristobal-Garcia M, et al. Uric acid-induced endothelial dysfunction is associated with mitochondrial alterations and decreased intracellular ATP concentrations. Nephron Exp Nephrol. 2012;121(3-4):e71-8. doi:10.1159/000345509

35. Prevention CfDCa. National Diabetes Statistics Reports 2020. Estimates of Diabetes and Its Burden in the United States. Department of Health and Human Services; 2020.

36. Yang T, Ding $\mathrm{X}$, Wang $\mathrm{YL}$, et al. Association between high-sensitivity C-reactive protein and hyperuricemia. Rheumatol Int. 2016;36:561-566. doi:10.1007/s00296-016-3429-z

37. Calle MC, Fernandez ML. Inflammation and type 2 diabetes. Diabetes Metab. 2012;38:183-191. doi:10.1016/j.diabet.2011.11.006

\section{Publish your work in this journal}

Diabetes, Metabolic Syndrome and Obesity: Targets and Therapy is an international, peer-reviewed open-access journal committed to the rapid publication of the latest laboratory and clinical findings in the fields of diabetes, metabolic syndrome and obesity research. Original research, review, case reports, hypothesis formation, expert opinion and commentaries are all considered for publication. The manuscript management system is completely online and includes a very quick and fair peer-review system, which is all easy to use. Visit http://www.dovepress.com/testimonials.php to read real quotes from published authors. 